\title{
Pour le meilleur et pour le pire : évolution des figures auctoriale et éditoriale au Québec
}

For Better or Worse: The Evolution of Authorial and Editorial Practices in Québec

\section{Para bien y para mal. Evolución de la figura autorial y editorial en Quebec}

\section{Marie-Pier Luneau}

Volume 51, numéro 2, avril-juin 2005

Les métiers du livre au Québec

URI : https://id.erudit.org/iderudit/1030088ar

DOI : https://doi.org/10.7202/1030088ar

Aller au sommaire du numéro

Éditeur(s)

Association pour l'avancement des sciences et des techniques de la documentation (ASTED)

ISSN

0315-2340 (imprimé)

2291-8949 (numérique)

Découvrir la revue

Citer cet article

Luneau, M.-P. (2005). Pour le meilleur et pour le pire : évolution des figures auctoriale et éditoriale au Québec. Documentation et bibliothèques, 51(2), 69-77. https://doi.org/10.7202/1030088ar
Résumé de l'article

C'est une lapalissade, l'éditeur tel qu'on le connaît aujourd'hui n'a pas toujours existé. Il fut un temps où l'auteur devait se charger lui-même des tâches réservées désormais à l'éditeur, notamment celles liées à la promotion du livre. Quel impact la consolidation de la fonction éditoriale a-t-elle eu sur la figure mythique de l'auteur? En observant d'un point de vue diachronique l'évolution des rapports entre auteurs et éditeurs au Québec du XIX ${ }^{\mathrm{e}}$ siècle à nos jours, cet article analyse en particulier le discours des auteurs sur la mise en marché de leurs livres.
Tous droits réservés (C) Association pour l'avancement des sciences et des techniques de la documentation (ASTED), 2005
Ce document est protégé par la loi sur le droit d'auteur. L’utilisation des services d’Érudit (y compris la reproduction) est assujettie à sa politique d'utilisation que vous pouvez consulter en ligne. 


\title{
Pour le meilleur et pour le pire: évolution des figures auctoriale et éditoriale au Québec
}

\author{
MARIE-PIER LUNEAU \\ Département des lettres et communications \\ Faculté des lettres et sciences humaines \\ Université de Sherbrooke \\ Marie-Pier.Luneau@usherbrooke.ca
}

\section{RÉSUMÉ | ABSTRACTS | RESUMEN}

C'est une lapalissade, l'éditeur tel qu'on le connaît aujourd'hui n'a pas toujours existé. Il fut un temps où l'auteur devait se charger lui-même des tâches réservées désormais à l'éditeur, notamment celles liées à la promotion du livre. Quel impact la consolidation de la fonction éditoriale a-t-elle eu sur la figure mythique de l'auteur? En observant d'un point de vue diachronique l'évolution des rapports entre auteurs et éditeurs au Québec du XIX ${ }^{e}$ siècle à nos jours, cet article analyse en particulier le discours des auteurs sur la mise en marché de leurs livres.

For Better or Worse: The Evolution of Authorial and Editorial Practices in Québec

It is a truism to say that the modern-day editor has not always been the person he has become today. There was a time when the author took on the role and responsibilities now assumed by the editor, namely those regarding the promotion of his book. What has been the impact of the consolidation of editing on the author? Taken from a diachronic perspective, the evolution of the relationship between authors and editors in Québec since the $19^{\text {th }}$ century is examined and as well as the position of authors regarding the marketing of their books.

\section{Para bien y para mal. Evolución de la figura autorial y editorial en Quebec}

Aunque parezca una perogrullada, el editor, tal como lo conocemos hoy, no siempre fue así. En cierto momento de la historia, el autor debió encargarse de las tareas reservadas al editor, especialmente las relacionadas con la promoción del libro. ¿Qué impacto tuvo la consolidación de la función editorial en la imagen mítica del autor? Dentro de un punto de vista diacrónico sobre la evolución de las relaciones entre los autores $y$ editores en Quebec desde el siglo XIX hasta nuestros días, este artículo analiza en especial el discurso de los autores sobre la comercialización de sus libros.
«Mais la vanité rôde. Car le jour où lécrivain porte son manuscrit à un éditeur pour que les lecteurs lui donnent vie, il n'est déjà plus un funambule. Il a perdu son innocence. Il change de registre. Il quitte le cirque pour entrer dans le merveilleux monde des "industries culturelles". Il peut songer même à prendre un agent. Il devient une petite entreprise.

La rencontre avec l'éditeur sera le dernier moment d'innocence. L'éditeur est face à l'auteur. Il sourit. Soupèse. Croit qu'il découvre ou fait naître un talent. Exit l'enfance et la virginité. Inévitablement le manuscrit sera usiné; traitement de texte, composition, titre, conclusion, normalisation, illustration de couverture, photo à l'endos du livre. Ah! la séance chez le photographe! On prend les mesures de l'auteur, l'écrivain s'efface. Publication, publicité, public.»

Jacques Godbout, «Regarde, regarde les arlequins!», in André Ricard et Jean-Guy Pilon (sous la direction de), Les Risques du métier, Montréal, l'Hexagone, 1991, p. 40.

A U CONTACT DE L'ÉDITEUR, l'auteur est expulsé du paradis virginal de la création pour entrer dans le vulgaire monde du commerce. Cette affirmation à l'emporte-pièce ne caricature qu'à peine les propos de Jacques Godbout cités en exergue. Une formule lapidaire qu'on trouve dans plusieurs livres portant sur les rapports entre auteurs et éditeurs résume le rôle concédé à l'éditeur dans l'élaboration de l'œuvre: "Les éditeurs boivent le champagne dans le crâne d'auteurs morts de faim» (Undseld, 1983: 14). Pour que le mythe du créateur désintéressé puisse opérer pleinement, l'éditeur doit endosser l'aspect impur lié à la vente du livre. Pierre Bourdieu a théorisé en long et en large la dénégation de l'économie, qui a entre autres pour étrange conséquence que, dans le domaine artistique, les succès pécuniaires soient considérés comme suspects alors que la pauvreté devient pour l'artiste un gage d'intégrité. Que ferait l'école, comment enseignerait-on la littérature sans l'image de l'auteur martyr - de Nelligan à Hubert 
Aquin -, de l'écrivain famélique n'ayant jamais touché un sou pour son génie, mais qu'on récompense une fois mort à coups d'encensoir dans les manuels scolaires? Pour passer à la postérité, l'auteur montre patte blanche, c'est-à-dire fait publiquement état soit de son indigence, soit de son dégoût pour le commerce, nonobstant sa réussite dans les faits - l'exemple de Godbout l'illustre à merveille. Qu'il soit impécunieux ou bien nanti en réalité, l'auteur rejette sur l'éditeur la responsabilité matérielle de l'activité intellectuelle.

Dans un article récent, Pascal Durand a très clairement dégagé ce rapport d'amour-haine qui lie les auteurs aux éditeurs. En posant comme prémisse que l'auteur et l'éditeur modernes émergent conjointement dans l'histoire, Durand énonce une observation fondamentale:

\section{"Tout semble en réalité se passer comme si l'éditeur, quelque avérée que soit la dualité de ses dispositions, constituait, autant que le par- tenaire, le bouc émissaire des auteurs, à la fois craint, respecté, adulé et vilipendé, conspué, haï, et comme s'il entrait dans ses fonctions, au sein d'un système où les intérêts commer- ciaux tendent désormais à se dissimuler der- rière un nuage de dénégations, de décharger les auteurs du poids coupable du calcul com- mercial [...].»}

Durand, 2002: 46-47

De toute évidence, il n'en a pas toujours été ainsi. Dans la perspective d'un numéro de revue consacré à l'évolution diachronique des métiers du livre, il m'est apparu pertinent de réfléchir aux transformations de la figure de l'auteur au Québec, en parallèle avec celle de l'éditeur. Bien que la question soit d'intérêt, il ne s'agit pas ici d'étudier comment le métier d'écrivain a, matériellement parlant, changé après la naissance de la fonction éditoriale au Québec. Sur le métier de l'écrivain au Québec, il existe en effet diverses enquêtes statistiques qui, mutatis mutandis, reconduisent les mêmes conclusions : Rares sont les écrivains qui vivent de leur plume; la plupart des auteurs habitent dans les grands centres urbains; ils exercent un second métier dans le domaine de l'enseignement; etc. Comme la problématique du numéro propose une réflexion sur les représentations liées aux métiers du livre, j'ai voulu plutôt m'intéresser à la façon dont l'apparition de la figure de l'éditeur a suscité la redéfinition de celle de lauteur et donc la transformation du discours de l'auteur sur sa propre pratique. On comprend bien, par exemple, quà partir du moment où un éditeur a pris en charge la production matérielle du livre, l'auteur a eu plus de temps à consacrer à l'écriture. Mais en renvoyant ainsi l'auteur à sa plume et en s'occupant de la mise en marché du livre l'éditeur a, volontairement ou non, endossé le costume du marchand et revêtu l'auteur de l'aura mystique du créateur. Si bien que de nos jours des auteurs-éditeurs comme Jacques
Godbout en arrivent à hiérarchiser les tâches: Ils sacralisent le travail de l'auteur et réduisent l'éditeur à un commerçant. Pourtant, avant qu'arrive ce nouveau personnage qu'est l'éditeur, l'auteur devait forcément assumer lui-même le travail de promotion du livre. Qu'en était-il alors de son rapport à l'argent, au négoce, à la publicité? Au moment où apparaît l'éditeur professionnel, comment se fait le transfert des responsabilités? L'auteur accepte-t-il de faire confiance à l'éditeur? De quelle façon apprend-il, progressivement, à entrer en partenariat symbolique avec l'éditeur, voire à se servir de celui-ci?

\section{QU'EST-CE QU'UN AUTEUR? QU'EST-CE QU'UN ÉDITEUR?}

Qu'est-ce qu'un éditeur? Pascal Durand (Durand, 2002) a posé la question sans détour en faisant écho au texte fondateur de Foucault "Qu'est-ce qu'un auteur?» (Foucault, 1969). Or, ces deux interrogations en apparence banales sont plus complexes qu'il n'y paraît. Placées dans un régime pragmatique, elles obtiendront des réponses simples, comme celles que fournissent les dictionnaires. L'auteur demeurera le seul créateur de l'œuvre littéraire, alors que l'éditeur sera, selon la formule traditionnelle, celui qui sélectionne, fabrique et diffuse le livre. Chacun son métier et les vaches seront bien gardées! La réalité semble toutefois infiniment plus complexe lorsqu'on étudie le rôle social de ces personnages. Le point commun entre ces deux fonctions reste la question de l'appropriation du texte. Tous les théoriciens s'entendent pour dire que l'auteur moderne n'apparaît que lorsqu'il revendique l'indissolubilité du lien l'unissant à son texte. Tant que l'auteur n'appose pas sa marque sur le texte, il n'existe pas, au sens moderne du terme. Pour revendiquer un rapport privilégié avec le texte, l'auteur utilisera divers moyens - de la signature plutôt que l'anonymat à lélaboration d'un style qui lui soit propre, en passant par la défense de ses droits devant les tribunaux. Or, on comprend aisément que l'auteur n'est pas né spontanément. Si la signature n'est qu'un exemple parmi d'autres de l'évolution du statut de l'auteur, elle reste cependant une excellente jauge, puisqu'il s'agit d'un signe d'appropriation irréfutable. Lucie Robert l'a noté: La signature, qui apparaît vers 186o, entraîne dans son sillon «une sorte de prestige individuel, la reconnaissance de l'artiste» (Robert, 1989: 52). C'est bien là la naissance de l'auteur moderne, accompagnée de celle de l'éditeur, car, comme le remarque Robert, à partir de 1860, apparaissent dans le paratexte non seulement le nom de l'auteur, mais aussi celui de l'éditeur. Bien qu'elles soient encore indéterminées, mouvantes, flottantes, les deux fonctions émergent conjointement.

Qu'est-ce encore qu'un éditeur? L'éditeur moderne sera celui qui, financièrement, moralement, intellectuellement, prendra le risque de la publication. Parce que mandaté par l'auteur comme intermédiaire 
entre lui-même et le public, l'éditeur est responsable de la publication. On pourra citer les exemples de précurseurs tels G.H. Cherrier, éditeur de Charles Guérin, ou encore l'abbé Casgrain, directeur de collections de livres de prix qui sélectionnait les manuscrits, se chargeait de les faire imprimer et de les distribuer dans les écoles. Casgrain prétendait d'ailleurs, dans ses lettres à Crémazie, s'être fait «l'éditeur» de celuici au Québec, alors que le poète était exilé en France. Or, l'émergence de la fonction éditoriale entraîne nécessairement une modification du statut de l'auteur. Pascal Durand constate par exemple, à la suite de Roger Chartier, que la transformation des rapports entre auteurs et libraires à la fin du XVIII ${ }^{\mathrm{e}}$ siècle amène en France le passage du modèle de l'écrivain dilettante, amateur d'art désintéressé refusant de tirer profit de son activité d'écriture, à celui de l'écrivain professionnel, pour qui les rétributions financières sont non seulement honorables mais indispensables puisqu'il aspire à vivre de sa plume. Selon l'ancien modèle, "l'amateurisme constituait même un insigne de distinction aristocratique» et l'écrivain attendait "du mécénat soutien financier en amont et reconnaissance sociale» (Durand, 2002: 26). C'est ce modèle d'écrivain qui prévaut encore au Québec au cours de la deuxième moitié du XIX ${ }^{\mathrm{e}}$ siècle. Maurice Lemire remarque en effet que ceux: «[...] qui voulaient consacrer une partie notable de leur carrière à l'écriture, loin de compter sur la vente de leurs livres sorientaient vers la fonction publique" (Lemire, 1983: 221). Ainsi, Lemire donne en exemple les Étienne Parent, Antoine Gérin-Lajoie, François-Xavier Garneau, Alfred Garneau, Pamphile Lemay, Joseph Marmette et Faucher de Saint-Maurice, qui, grâce à cette forme de mécénat déguisé, pouvaient ainsi «taquiner la muse à l'abri de la faim» (Lemire, 1983: 222). Le fait que ce modèle d'écrivain soumis au mécénat (en l'occurrence, celui de l'État) précède, dans l'histoire, le modèle de l'écrivain professionnel n'est sans doute pas étranger à toute la suspicion qu'inspirera par la suite l'argent dans le monde des lettres. En évoquant le cas de Ludger Duvernay, pour qui écrire n'est pas un métier mais tient de l'engagement social et patriotique, Daniel Mativat proclame que cette:

\section{«[...] vision du rôle de lécrivain dans la société, [...] aura de nombreux adeptes et contribuera elle aussi à renforcer un des préjugés culturels québécois les plus tenaces: celui de la gratuité de l'art et de l'embrigadement obligatoire $d u$ créateur au service de la cause nationale.»}

Mativat, 1996: 194.

Les échanges épistolaires entre Casgrain, "éditeur» de Crémazie, et l'auteur du Drapeau de Carillon sont symptomatiques de l'amorce d'une mutation dans le monde du livre. De l'extérieur, Crémazie dispose du recul nécessaire pour évaluer la situation de l'écrivain au Québec. N'écrit-il pas à Casgrain, en 1866:

«Puisque tout travail mérite un salaire, il faut donc que l'écrivain trouve dans le produit de ses veilles, sinon la fortune, du moins le morceau de pain nécessaire à sa subsistance. Autrement vous n'aurez que des écrivains amateurs. »

Crémazie, 1976: 76 .

Le jugement jette l'anathème sur le modèle de l'écrivain qui occupe une sinécure et se fait un point d'honneur de dédaigner les profits de ses livres. L'écrivain professionnel voudra au contraire faire de son activité un métier et défendre ses droits. Cela ne veut pourtant pas dire qu'il mettra au rancart l'idée de la gratuité de l'art, puisque l'éditeur pourra lui servir de garant négatif, autorisant les dénégations économiques. Ce bouleversement, visiblement amorcé à la fin du XIX ${ }^{\mathrm{e}}$ siècle, s'accélère au tournant du $\mathrm{XX}^{\mathrm{e}}$ et se cristallise dans les années 1920 et 1930.

La période de l'entre-deux-guerres sert de cadre à l'établissement de l'éditeur et de l'écrivain professionnels. Édouard Garand, Albert Lévesque, Albert Pelletier, Louis Carrier et Eugène Achard sont autant d'emblèmes de la professionnalisation du métier d'éditeur, dissocié de l'imprimerie et de la librairie, pleinement responsable de la publication et non plus de manière épisodique ou à la pièce, comme c'était le cas au XIX ${ }^{\mathrm{e}}$ siècle. Certes, dans les années 1920 et 1930, l'éditeur assure la sélection, la fabrication, la diffusion de l'œuvre mais, par-delà ces tâches professionnelles, il assume également une fonction représentative, comme au théâtre. Il devient lui aussi une figure, au même titre que l'auteur. Quand, par exemple, en 1928, Simone Routier conseille à Alfred DesRochers d'adresser de ses poèmes à chaque quotidien du Canada pour publiciser L'Offrande aux vierges folles qu'il vient d'éditer lui-même, elle effleure le sujet:

«Voyez-vous lorsqu'on ne peut s'offrir les luxueuses éditions du Mercure (on me demandait $450 \$$ ou 350 \$ plus $25 \%$ sur chaque livre, lancement du livre compris) qui font le lancement du livre et en imposent la critique aux journaux (au détriment de ces derniers, d'ailleurs), il faut bien s'aider un peu.»

Routier in Brosseau, 1998: 91.

L'auto-édition est moins onéreuse, mais assortie d'un capital symbolique médiocre, en comparaison avec le prestige que procure à l'auteur le label éditorial. L'auteur qui s'auto-édite devra faire des efforts pour combler le manque à gagner. C'est dire que la figure de l'éditeur s'impose maintenant comme représentation symbolique, au-delà de ses activités matérielles. C'est presque un cliché, mais néanmoins vrai: désormais, l'auteur signe le texte, l'éditeur signe le livre. 


\section{À l'instar de l'auteur du XIX'e siècle, Groulx se charge de trouver lui-même un imprimeur, en l'occur- rence L'Action sociale, et paie les frais liés à l'impression.

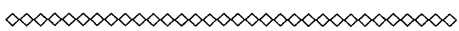

Pour mesurer l'évolution des figures d'auteur en rapport avec celles des figures d'éditeur au Québec, il m'est apparu opportun d'observer la trajectoire de trois écrivains majeurs, comme autant de ponctions nous permettant de tirer des conclusions synchroniques et diachroniques. Lionel Groulx, Alfred DesRochers et Gabrielle Roy appartiennent à trois générations d'écrivains qui ont justement vu apparaître, se fragiliser puis se solidifier la figure de l'éditeur au Québec, tout au long du $\mathrm{XX}^{\mathrm{e}}$ siècle.

\section{LIONEL GROULX, MARCHAND DE RÊVES ET DE PROSE}

Dans leurs mémoires, les écrivains ont tendance à malmener les éditeurs, les tenant invariablement responsables de leurs échecs, mais jamais de leurs succès. Lionel Groulx ne déroge pas à la règle. Dans ses souvenirs, ses principaux éditeurs subissent un traitement peu glorieux - comme bien d'autres de ses contemporains, au demeurant. Groulx ne se gêne pas pour citer de longs extraits d'une lettre d'Henri D’Arles dans laquelle Albert Lévesque est décrit comme «un jeune guerrier, un patron de boutique, envahisseur et encombrant» (Groulx, 1971: 379). Non seulement Groulx donne-t-il raison à D'Arles, mais il attribue à Lévesque la responsabilité de l'échec de L'Action française, lui imputant une faillite, l'accusant de surcroît de ne pas lui avoir versé tous ses droits d'auteur. La coupe est pleine et l'insulte amène l'éditeur à rédiger une «Rectification aux mémoires de Groulx", qui ne sera publiée qu'en 1994 (Michon, 1994: 115-130). Quant à Eugène Achard, des Éditions du Zodiaque, il apparaît sous la plume du mémorialiste comme un véritable enquiquineur. Groulx n'aime pas "gratter ses fonds de tiroir», mais il le fera pourtant, car il lui faut «hélas [...] compter parfois avec la gent tenace des éditeurs. Et le plus sûr moyen de se délivrer de ces crampons, ne serait-ce pas de céder, en définitive, à leur importunité» (Groulx, 1972: 226). L'éditeur n'a-t-il d'autre fonction que celle de la mouche du coche? Adieu, les titres d'accoucheur, de père spirituel, d'époux fidèle dont on coiffe parfois l'éditeur!

On peut comprendre que Lionel Groulx se méfie de ce nouveau venu dans le monde du livre et c'est Albert Lévesque qui en paiera le plus haut prix. Lionel Groulx commence à publier en 1912, alors que le métier d'éditeur, au sens moderne du terme, n'est pas encore solidement implanté. À l'instar de l'auteur du XIX ${ }^{\mathrm{e}}$ siècle, Groulx se charge de trouver lui-même un imprimeur, en l'occurrence L'Action sociale, et paie les frais liés à l'impression. Ses livres suivants seront imprimés au Devoir, puis à la Bibliothèque de l'Action française, au début des années 1920. À l'arrivée d'Albert Lévesque dans la maison en 1926, Groulx connaît bien les rudiments des métiers d'auteur et d'éditeur, puisqu'il s'est toujours chargé de la production et de la diffusion de ses livres - allant jusquà prendre des pseudonymes pour faire l'éloge de ses propres publications dans la revue L'Action française. Groulx dispose également d'un réseau d'amis propagandistes rejoignant les quatre coins du Québec, puissant au point qu'Albert Lévesque finit par admettre, dans une lettre du 5 juillet 1933:

"Quel que soit l'éditeur que vous choisirez, aucun ne pourra déployer assez d'énergie et d'influence pour remplacer celles dont vous disposez vous-même pour assurer le succès de vos ouvrages. Je me réjouis du succès de votre dernier roman, mais je constate à ma propre satisfaction qu'il n'est pas dî̀ à votre éditeur, mais à vous-même, non seulement à cause de votre réputation de premier écrivain de l'heure au Canada français, mais parce que vous vous en êtes constitué le principal propagandiste.»

Lévesque in Luneau, 2003: 138.

Au moment où paraissent les premières œuvres de Groulx, le modèle de l'éditeur indépendant n'existe pas. Les fonctions auctoriale et éditoriale sont liées comme les deux côtés d'une même médaille et Groulx les assume avec brio - à preuve les ventes fulgurantes des Rapaillages et de L'Appel de la race. Pourquoi feraitil confiance à l'éditeur, qui s'immisce littéralement entre lui et son public? Pas étonnant qu'après avoir quitté Lévesque, Groulx confie plutôt ses manuscrits à un libraire-imprimeur, Granger. Voilà une bonne façon de reprendre le contrôle. Pourtant, encore une fois Lionel Groulx sera déçu. Arrive un moment où Granger réimprime ses livres sans son consentement, quittant son rôle de simple exécutant des ordres de l'auteur pour prendre des décisions de nature éditoriale.

Avec la Seconde Guerre mondiale est venu «le temps des éditeurs ». Le deuxième volume de L'Histoire de l'édition littéraire au Québec montre bien que le conflit mondial fait souffler sur l'édition canadiennefrançaise un vent inespéré. Comme le note Jacques Michon, les "arrêtés en conseil sur les brevets, le droit d'auteur et les marques de commerce adoptés en septembre et octobre 1939 permettent aux éditeurs canadiens de reproduire, conformément à la loi, tous les ouvrages publiés en territoire ennemi.» (Michon, 2004: 23) 
Or, à compter de juin 1940, la France devient «territoire ennemi». Les éditeurs canadiens peuvent donc réimprimer à loisir les titres français, moyennant le versement d'une redevance de $10 \%$ au bureau du Séquestre des biens ennemis. Apparaît le personnage de l'éditeur puissant qui négocie d'égal à égal avec ses confrères européens et a ses entrées un peu partout dans la francophonie - Fides installe même une succursale au Brésil! Dans un étrange renversement des rôles, la figure mythique de l'éditeur devient plus grande que celle de l'auteur. Un commentateur de l'époque, Pierre Baillargeon, en dresse le portrait:

"Léditeur publie un million de livres. Il fait de bonnes affaires: l'enseignement obligatoire lui a donné un public; la publicité a remplacé la critique. Il n'a plus besoin des salons, des académies, des scènes. Il n'a même plus besoin d'écrivains célèbres, parce qu'il devient plus considérable qu'eux. Il devient ce que sont devenus les chefs d'orchestre, qui éclipsent les compositeurs. Comme on est attiré plus par un Toscanini que par Beethoven, que par Bach, de même on achète les livres d'un éditeur sans regarder leurs signatures. La signature se confond avec le titre, et le nom de l'éditeur passe pour la marque de fabrique."

Baillargeon in Michon, 2004: 80.

Après la guerre, Groulx n'a donc d'autre choix que d'entrer, pour la première fois, en partenariat symbolique avec l'éditeur. La prophétie d'Albert Lévesque décrivant un Groulx plus puissant que ses éditeurs ne tient plus. Devant cette transformation du champ, Groulx est contraint de changer de discours. Au début du siècle, il n'avait aucun scrupule à tenter de vendre ses livres auprès d'institutions ou d'amis bien placés. Il parlait «affaires» dans ses lettres, consentant des rabais à ses meilleurs «agents de propagande», une expression qu'il utilisait pour désigner son ami Wilfrid Lebon. Or, plus la figure de léditeur se précise, plus il devient gênant pour un créateur de s'afficher marchand de prose. L'auteur, noble et désintéressé, est certes un marchand de rêves mais, pour paraphraser Matyssens, il ne doit pas en vivre! En 1949, Groulx insiste pour qu'on lui verse des droits sur la réédition d'une de ses conférences, tout en affirmant que cette exigence ne doit pas empêcher le projet de réédition. Vis-à-vis du public, l'image du créateur éthéré doit supplanter tout le reste. "J'entends rester un auteur et ne pas devenir un marchand", écrit-il à Dominique Beaudin le 31 juillet 1949 (Groulx in Luneau, 2003: 171). En 1950, Groulx publie à l'Action nationale son œuvre maîtresse, L'Histoire du Canada français. L'historien, couvert de récompenses, atteint le statut de mythe. Lauteur charismatique se dégage alors de toute responsabilité matérielle auprès de ses amis, comme le prouve cette lettre à Antonin Lamarche datée du 13 décembre 1950 :
Or, plus la figure de l'éditeur se précise, plus il devient gênant pour un créateur de s'afficher marchand de prose. L'auteur, noble et désintéressé, est certes un marchand de rêves mais, pour paraphraser Matyssens, il ne doit pas en vivre!

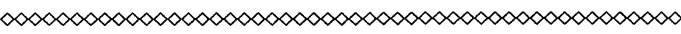

"Je vous remercie de ne pas m'avoir tenu responsable de la publicité tapageuse faite autour de mon dernier ouvrage. Que voulezvous? Le coût d'impression est devenu prohibitif ou presque, en notre pays. Il est bien difficile d'empêcher un éditeur de se reprendre en tapant fort sur l'esprit et même sur les nerfs $d u$ public, surtout lorsqu'il porte, lui seul, tous les frais de l'édition.»

Groulx in Luneau, 2003: 173.

Si dans les faits l'auteur continue de poser des gestes concrets pour diffuser et vendre ses livres, nulle trace ne doit en rester car le sage tient à son indépendance. Quel contraste avec le jeune auteur-éditeur du début du siècle qui avait envoyé une lettre circulaire à tous les directeurs de collège pour prendre leurs commandes à l'avance avant de réimprimer Les Rapaillages! Après la guerre, la figure de l'éditeur est devenue à ce point importante que Groulx ne peut continuer de la contourner. À son corps défendant, il a formé un tandem obligé avec cette créature étrange, qui lui permettra à tout le moins de pratiquer la dénégation de l'économie. À Benoît Lacroix, qui s'inquiète de la lenteur avec laquelle Fides prépare la publication de son «Lionel Groulx» dans la collection «Classiques canadiens », il rétorque, le 15 mars 1967:

"On a déjà dit de cette maison qu'elle était "le cimetière des manuscrits." Je ne serai pas aussi sévère. Mais ce sont des commerçants. Ils ont d'autres chats à soigner.»

Groulx in Luneau, 2001: 335 .

\section{ALFRED DESROCHERS, PROFESSION : ÉCRIVAIN}

Richard Giguère a déjà déjà dressé un portrait éloquent et fort documenté des rapports entre Alfred DesRochers et ses éditeurs, des années 1930 aux années 1960 (Giguère, 1994). Il ne s'agit pas ici de reproduire ses conclusions, mais plutôt de voir comment DesRochers se positionne par rapport à la figure de l'éditeur en comparaison avec un écrivain de la génération précédente, tel Lionel Groulx. D'entrée de jeu, des différences sont manifestes. Alors qu'à cette époque Lionel Groulx avait une importante expérience 
en la matière, DesRochers arrive à la publication au moment même où se professionnalise le métier d'éditeur. Il choisit cependant lui aussi l'auto-édition pour son premier recueil, L'Offrande aux vierges folles. Comme le note Richard Giguère, "choisir» est un bien grand mot. Contrairement à Lionel Groulx, DesRochers n'est pas branché sur le milieu de l'édition montréalaise. Dans la mesure où DesRochers est inconnu, habite Sherbrooke et publie de surcroît de la poésie, genre qui rejoint peu de lecteurs, l'auto-édition devient pour lui "une nécessité plus qu'un choix» (Giguère, 1994: 14). Dès l'automne 1929, toutefois, Albert Lévesque offre à DesRochers de publier À l'ombre de l'Orford. Malgré les conseils de Louis Dantin d'accepter la proposition, DesRochers préfère encore l'auto-édition. Ce n'est qu'à la suite de négociations musclées que Lévesque et DesRochers parviennent à s'entendre, Lévesque ayant consenti de multiples concessions à l'auteur. Ici, on ne sent pas, comme chez Groulx, la méfiance de l'auteur envers l'éditeur. DesRochers semble pleinement conscient de la valeur économique du travail de l'écrivain et est déterminé à se tailler la meilleure part du gâteau. Pas de doléances larmoyantes ni de récriminations contre les «éditeurs-marchands", simplement l'affirmation d'un écrivain professionnel qui vise à tirer profit de son activité devant un éditeur professionnel. Il n'en résulte pas pour autant des rapports pleinement sereins entre les deux protagonistes, mais l'entrée en partenariat symbolique avec l'éditeur se produit beaucoup plus rapidement que chez Lionel Groulx. L'auteur de L'Appel de la race attend l'après-guerre pour collaborer vraiment avec ses éditeurs; DesRochers le fait quant à lui dès 1930, mais en ne cédant sur rien. L'écrivain sait qu'il a besoin de l'éditeur et en prend son parti plutôt que de ramer à contre-courant:

"Rédige-moi un contrat pour Paragraphes qui me permettra de faire au moins $50 \$$, et je te le signerai. Ou si tu préfères, assure-moi d'un chèque de $50 \$$ et je te vendrai au moins 100 exemplaires au gouvernement. »

DesRochers in Giguère, 1994: 16.

C'est d'une collaboration réelle qu'il s'agit ici, d'un travail d'équipe qui n'exclut pas cependant les grincements de dents. Avec DesRochers - on pourrait sans doute étendre ce constat à plusieurs écrivains de la jeune génération comme Jean-Charles Harvey, Éva Senécal et Simone Routier, très conscients des règles qui gouvernent le champ littéraire-, on bascule manifestement vers le modèle de l'écrivain professionnel qui souhaite vivre de sa plume et n'éprouve aucune honte à le dire. Conscient de ses ressources, DesRochers consulte même en 1947 l'avocat de la Société des écrivains canadiens pour dénouer l'impasse éditoriale qui le lie à la Société des éditions Pascal. L'image qui se dégage de ses rapports avec ses éditeurs n'est pas celle d'une victime. Elle évoque plutôt un interminable bras de fer. En ce sens, Richard Giguère a tout à fait raison d'affirmer:

"[...] le mythe qui ne tient plus avec Alfred DesRochers - il faudrait voir ce qu'il en est pour d'autres écrivains de son époque - est celui du poète qui a la tête dans les nuages et qui ne s'occupe pas des conditions d'édition et de diffusion de ses livres. DesRochers lit ses contrats de près et les négocie de façon très serrée, il exige des changements à de nombreux articles ou alors réécrit les contrats d'un bout à lautre, car il vise toujours les meilleures conditions et les meilleurs bénéfices possibles.»

Giguère, 1994: 22.

\section{GABRIELLE ROY, LA CRÉATRICE}

Groulx a publié son premier livre dans les années 1910, DesRochers, à la fin des années 1920. Gabrielle Roy quant à elle, arrive à l'édition en 1944. Quelques années seulement ont passé depuis la première publication de L'Offrande aux vierges folles et pourtant le monde du livre - la société, en fait - a complètement changé de visage. Entre les deux, une guerre mondiale est passée. Contrairement à Lionel Groulx et à Alfred DesRochers, Gabrielle Roy ne songe pas à l'auto-édition après avoir écrit son premier roman, Bonheur d'occasion. Elle s'adresse à Gérard Dagenais, propriétaire de la Société des éditions Pascal, une de ces entreprises nées pendant la Seconde Guerre mondiale. C'est dire encore une fois que la figure de l'éditeur professionnel a pris du galon auprès des auteurs depuis le début du siècle. À bon droit, Gabrielle Roy sera toutefois vite désenchantée par les services de son éditeur. Aucune des clauses du contrat signé entre les deux parties n'est respectée: Le livre paraît neuf mois après la date de publication prévue, il est publié en deux volumes plutôt qu'un seul comme le voulait l'auteure et, malgré les ventes impressionnantes du roman, l'éditeur remet à Gabrielle Roy des chèques sans provision. Rien d'étonnant au fait que la romancière ait recours à un adjuvant, en l'occurrence Jean-Marie Nadeau, qui deviendra, selon François Ricard, «l'homme d'affaires» de Gabrielle Roy:

«Elle s'en remet à lui pour la négociation et parfois même la signature des contrats, la perception de ses redevances, la vente de ses textes à l'étranger, ses rapports avec le fisc et, de manière générale, tout ce qui concerne l'administration de ses droits et de son argent.»

Ricard, 1996: 269.

Décidée à ne pas laisser le succès de Bonheur d'occasion lui filer entre les doigts à cause de l'incompétence de son éditeur, Gabrielle Roy, grâce à Nadeau, reprend ses droits sur le roman. À l'avenir, Dagenais 
n'agit plus quà titre de distributeur du livre. À partir de 1946, après la disparition de la Société des Éditions Pascal, Beauchemin reprend la distribution, l'écrivaine demeurant encore le maitre-d'œuvre de la publication. Peut-on voir ici le juste retour de l'ancien modèle de l'auto-édition? Pas tout à fait, car la forme d'auto-édition empruntée par Roy ne ressemble qu'en apparence à celle que pratiquaient Groulx et DesRochers. En réalité, Jean-Marie Nadeau négocie le prêt financier nécessaire pour l'impression des livres; c'est même lui qui s'occupe de les entreposer. C'est aussi grâce à Nadeau que Bonheur d'occasion devient un succès international. Celui-ci décroche le contrat avec la firme américaine Reynal \& Hitchcock. Le roman sera alors vendu au plus vieux club de livres américain, la Literary Guild of America, on l'imprimera à 700 ooo exemplaires et les droits seront même cédés à la Universal Pictures! Nadeau fait aussi connaitre Bonheur d'occasion aux éditions Flammarion en France, ce qui permettra au roman de décrocher le prix Femina en 1947. De toute évidence, un succès aussi retentissant ne peut se gérer sans la présence d'un homme d'affaires. Fait plus intéressant, dès le début de sa carrière, Gabrielle Roy donne l'impression de ne pas s'intéresser à l'aspect économique de son travail. Or, François Ricard l'a très bien démontré, si Gabrielle Roy est sincère lorsqu'elle affirme que la publicité et la promotion lui pèsent,

\begin{abstract}
«[...] cela ne l'empêche pas de s'occuper activement de ses affaires et dêtre à la fois étonnée et ravie par la popularité de Bonheur d'occasion et par les éloges de la critique. N'aspire-t-elle pas depuis toujours à la notoriété, d̀ l'admiration d'autrui, à la fortune? Loin de se désintéresser du succès de son livre, elle tient à en recueillir les bénéfices, surtout financiers, et se montre jalouse de ses droits, encourageant constamment $M^{e}$ Nadeau à faire preuve de la plus grande rigueur dans ses négociations et son administration.»
\end{abstract}

$$
\text { Ricard, 1996: } 275 \text {. }
$$

Ainsi, comme Lionel Groulx à peu près à la même époque, Gabrielle Roy développe un double discours quant à son travail d'écrivaine. Lorsque la radio de CKAC de Montréal lui offre d'adapter Bonheur d'occasion en feuilleton, elle refuse, arguant d'une part le manque de temps nécessaire à la révision des textes, mais pratiquant aussi la dénégation de l'économie: "J'éprouve une extrême répugnance à prêter mon nom à une série d'émissions commanditées. » (Roy in Ricard, 1996: 279). Qu'on se le tienne pour dit, l'écrivain mythique est un être libre dont la pureté se corroderait en effleurant la publicité. Gabrielle Roy tourne même en dérision la figure de l'éditeur Flammarion dans un texte humoristique intitulé «Comment j'ai reçu le prix Femina». L'auteure y apparaît comme la victime d'un tourbillon médiatique contrôlé presque

\section{Gabrielle tient à garder sa pleine liberté à l'égard de Stanké, bien décidée à ne plus jamais se retrouver dans la position où l'ont placée jadis ses relations avec Flammarion.}

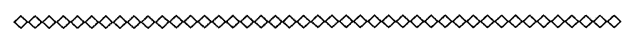

machiavéliquement par des membres de jury superficiels et mondains. Le prix a lui aussi un prix: Lauteure apprend que ce Femina s'achète, si l'on accepte «de faire quelques visites...» (Roy, 1978: 180). Dans toute cette mascarade, le plus grand coupable reste l'éditeur qui joue le jeu à fond. Lorsque, pendant la course, l'écrivaine tombe malade, l'éditeur l'invite froidement «à survivre au moins jusqu'à l'attribution du prix»" (Roy, 1978: 183).

Chez Gabrielle Roy, le monde du commerce se dissocie d'emblée de l'activité créatrice. Dans les années 1970, à une époque où elle exerce un contrôle très serré sur l'édition de ses livres - elle négocie notamment la rétrocession des droits avec Hartcourt Brace et Flammarion et change aussi d'éditeur au Québec -, Roy en arrive à déclarer: "J'ai découvert ce qui a été vrai pendant la majeure partie de ma vie et est devenu de plus en plus évident pour moi dernièrement, le fait que je m'inquiète très peu de l'argent.» (Roy in Ricard, 1996: 499), une affirmation paradoxale s'il en est car, comme le note François Ricard, cela «[...] ne l'empêche pas, à cette époque comme depuis le début de sa carrière, de surveiller de très près l'administration de ses finances, de réclamer le paiement de ses redevances à chaque échéance, et de ne jamais dépenser plus que nécessaire, comme si l'argent lui était compté. » (Ricard, 1996: 499).

Ici, la dichotomie entre les activités créatrice et économique n'entrave pas le partenariat entre l'auteur et l'éditeur, bien au contraire. L'éditeur lui-même se fera le héraut de l'écrivain mythique, participant ainsi à la diffusion d'une image sacralisée. À la fin du parcours de Gabrielle Roy, Alain Stanké remplit ce rôle, quoi qu'en dise par ailleurs François Ricard. D'une part, la biographie de Roy, signée par Ricard, prétend que dans les années 1970 Gabrielle Roy refuse de se laisser attacher à un éditeur: "Gabrielle tient à garder sa pleine liberté à l'égard de Stanké, bien décidée à ne plus jamais se retrouver dans la position où l'ont placée jadis ses relations avec Flammarion.» (Ricard, 1996: 496-497). D'autre part, Stanké est catégorique:

\section{"[...] pour elle, exercer son métier d'écrivain ne pouvait se faire que dans un contexte de sympathie et de fidélité. La nécessité donc de collaborer avec un seul éditeur - son éditeur - était une loi. [...] Je fus celui-là avec cons-}


tance et plaisir durant les 10 dernières années de sa vie.»

Stanké, 1999: 50.

Il faut bien dire que la correspondance de Gabrielle Roy donne raison à Stanké. Même si elle le tient à l'œil et le rappelle occasionnellement à l'ordre, l'écrivaine lui demande textuellement d'être son "protecteur" (Roy in Toussaint, 2004: 279). Qui plus est, sa volonté d'établir avec quelques éditeurs un rapport de "fidélité» apparaît clairement dans une lettre datée du $1^{\text {er }}$ septembre 1977, alors que Roy écrit à Stanké:

«[...] j’attends la visite de mon éditeur de Toronto, Jack McClelland, qui, lui, attend son tour pour venir depuis quelques années. [...] De tous les éditeurs que j'ai eus, il reste le seul avec qui je suis demeurée depuis le début n'ayant jamais eu avec lui le moindre ennui, le moindre désaccord. Je souhaite que nous en arrivions vous et moi à la même harmonieuse entente, mais, convenez-en, il faut un peu de temps pour cela.»

Roy in Toussaint, 2004: 281-282.

De fil en aiguille, Alain Stanké devient le «filtre» qui protège l'écrivaine du reste du monde. Quelle belle illustration de la séparation entre les fonctions auctoriale et éditoriale... Peu importe la réalité, l'éditeur et l'auteur diffusent conjointement dans leurs discours des représentations précises de ces deux figures. Ainsi, le créateur pur crée, dégagé des soucis matériels, protégé des invasions de tout acabit, alors que l'éditeur, marchand, s'occupe de la cuisine:

«Comme elle ne savait pas prononcer le mot non [...] et qu'elle ne voulait jamais dire oui, je devins rapidement son porte-parole. Celui qui refusait en son nom les interviews, conférences, présidences d'honneur et autres propositions, des plus alléchantes aux plus farfelues. [...] Une ouvre se suffit à elle-même, avait-elle coutume de dire. Elle a sa propre vie. Dès qu'un livre est né, il n'appartient plus à l'auteur!»

Stanké, 1999: 50.

\section{CONCLUSION}

C'est le rêve de tout écrivain: créer uniquement, ne faire rien d'autre. Gabrielle Roy s'en est approchée, pouvant consacrer sa vie à l'écriture puisqu'elle vivait de sa plume. Et alors? Ce songe tient du mirage, puisqu'elle n'y est jamais parvenue complètement. Ainsi le concède cette lettre adressée à Margaret Laurence:

«J'ai le sentiment que vous et moi avons été usées beaucoup plus par les lettres d'affaires et toutes les requêtes qu'on nous fait que par notre propre travail.
Il me semble qu'il a dû avoir un temps où les écrivains écrivaient leurs livres et qu'ensuite on les laissait en paix.» (Roy in Ricard, 1996: 492)

N'en déplaise à Gabrielle Roy, ce temps idyllique n'est jamais venu, pas plus à l'époque de Crémazie, où l'on pestait contre l'incompétence des typographes ou contre la voracité des imprimeurs, que de nos jours, alors que le cliché de l'éditeur carnassier reste très présent dans le monde du livre. Peut-il en être autrement, dans la mesure où les fonctions éditoriales et auctoriales ont séparé leurs chemins pour suivre des routes parallèles?

Au cours du $\mathrm{XX}^{\mathrm{e}}$ siècle, auteurs et éditeurs ont conclu un partenariat symbolique pour défendre une cause commune: faire lire, faire vendre des livres. Au moment où Lionel Groulx fait son entrée dans le monde de la publication, l'éditeur professionnel demeure un cas d'espèce. C'est certainement cet auteur qui a le plus de difficulté à apprivoiser le nouveau venu, contrairement à un Alfred DesRochers qui, somme toute, tire bien son épingle du jeu. Quant à Gabrielle Roy, elle est prête au départ à confier ses manuscrits à un éditeur, toute à sa joie de pouvoir simplement écrire. Malheureusement, elle apprend à ses dépens qu'elle ne peut se délester des tâches matérielles liées à son métier d'écrivaine. Sa carrière durant, elle surveille de près ses éditeurs, bien que cette tâche l'horripile. Simultanément, elle adopte l'image de la créatrice dégagée du poids commercial de l'œuvre et l'éditeur la seconde dans la diffusion de cette image.

Reste une évidence: l'écrivain rêve de se consacrer tout entier à son œuvre, tout en voulant être lu, ce qui suppose qu'il s'intéresse aussi à la diffusion. Voilà pourtant la face cachée de l'activité littéraire car, dans la mesure où l'éditeur existe, on aime à croire qu'il pourra endosser à lui seul les fonctions qu'on dit impures. Dans le champ littéraire moderne, cela fait implicitement partie des tâches de l'éditeur, dépassant donc la sélection, la fabrication et la distribution du livre, pour atteindre le niveau symbolique de la représentation. Comme le notait Pascal Durand, léditeur se transforme en bouc émissaire pour les auteurs. Dans les années 1960, Fernand Dumont et Jean-Charles Falardeau ont mené une enquête sur le statut de l'écrivain au Québec. On ne sourcillera pas devant une des conclusions concernant les rapports auteurséditeurs :

«Dans l'ensemble, les écrivains nous ont paru assez durs pour les éditeurs. "Ils sont assez gentils avec nous, dit-on, mais on ne croit pas que l'éditeur puisse aider l'écrivain à se créer un public. "[...] Pour plusieurs écrivains, l'éditeur est uniquement un homme d'affaires, un commerçant.»

Dumont et Falardeau, 1964: 88-89. 
Contre mauvaise fortune, l'éditeur fait bon cœur et laisse dire qu'il n'est qu'un marchand. Pour que le public continue de croire à la suprématie du créateur sur l'œuvre, l'auteur doit se maintenir sur son piédestal. Qu'on ait proclamé sa mort dans les années 1960 n'y a rien changé. C'est une des conclusions que tire Laurence Santantonios de sa récente enquête auprès d'éditeurs français:

«Curieux comme un éditeur interrogé aujourd'hui sur sa relation aux auteurs et à la littérature minimise son rôle, s'efface derrière le créateur qui le fascine et l'agace tout à la fois! Comme s'il méconnaissait son pouvoir de dire oui, de dire non, de choisir le titre, de couper un chapitre, d'encourager un auteur, de l'étouffer dans l'oeuf. Lorsque je leur demande: "Avez-vous de l'influence sur vos auteurs?" ils répondent: "Oh non, surtout pas, ce serait catastrophique, je ne suis qu'un passeur, qu'un intermédiaire".»

Santantonios, 2000: 255 .

En définitive, le plus grand talent que puisse avoir l'éditeur moderne ne réside-t-il pas dans sa capacité à jouer les boucs émissaires, notamment en se faisant plus petit que l'auteur charismatique? La reine Zabo, patronne des éditions du Talion dans le roman $L a$ Petite Marchande de prose de Daniel Pennac, le croit suffisamment pour en faire un poste officiel, qu'elle confie bien sûr à Benjamin Malaussène. On peut dire que c'est avec verve que Sa Majesté la reine Zabo résume ce qu'on attend, aujourd'hui, d'un éditeur:

"Écoutez, Malaussène, je vous ai engagé comme bouc émissaire pour que vous vous fassiez engueuler à ma place, pour que vous épongiez les emmerdes en pleurant au bon moment, pour que vous résolviez l'insoluble en ouvrant grand vos bras de martyr, en un mot, pour que vous endossiez. Or, vous endossez formidablement. Vous êtes un endosseur de première [...].»

Pennac, 1989: 28. $(-$

\section{SOURCES CONSULTÉFS}

Brosseau, Marie-Claude. 1998. Trois Écrivaines de l'entre-deuxguerres : Alice Lemieux, Éva Senécal et Simone Routier. Québec, Nota Bene. 126 p.

Crémazie, Octave. 1976. Euvres complètes, vol. II. Prose. Texte établi par Odette Condemine. Ottawa, Éditions de l'Université d'Ottawa. 438 p.
Dumont, Fernand et Falardeau, Jean-Charles. 1964. Littérature et Société canadiennes-françaises. Québec, Presses de.l'Université Laval. $272 \mathrm{p}$.

Durand, Pascal. 2002. Qu'est-ce qu'un éditeur? Texte $\mathrm{n}^{\mathrm{os}} 31 / 32$, p. 13-55.

Foucault, Michel. 1969. "Qu'est-ce qu'un auteur?», Bulletin de la Société française de philosophie, LxIV, p. 73-104.

Giguère, Richard. 1994. "Alfred DesRochers et ses éditeurs : des relations d'affaires tendues». In Michon, Jacques (sous la direction de). L'Édition littéraire en quête d'autonomie. Albert. Lévesque et son temps, Québec, Presses de l'Université Laval, p.13-24.

Giguère, Richard. 1993. In Benoît Melançon et Pierre Popovic. Les Facultés des lettres. Recherches récentes sur l'épistolaire québécois, Département d'études françaises, Université de Montréal, p. 163-181.

Groulx, Lionel. 1970-1974. Mes mémoires. Tomes 1 à 4. Montréal, Fides.

Lemire, Maurice. 1983. «Les relations entre écrivains et éditeurs au Québec au XIX ${ }^{\mathrm{e}}$ siècle». In Yvan Lamonde (sous la direction de). L'Imprimé au Québec. Aspects historiques (18 ${ }^{e}-20^{e} \mathrm{~s}$.), Québec, IQRC, p. 208-224.

Luneau, Marie-Pier. 2003. Lionel Groulx. Le mythe du berger. Montréal, Leméac. $226 \mathrm{p}$.

Luneau, Marie-Pier. 2001. Le Manche et la Cognée: Lionel Groulx, une vie d'écritures. Thèse de doctorat en études françaises, Université de Sherbrooke. $486 \mathrm{p}$.

Mativat, Daniel. 1996. Le Métier d'écrivain au Québec (1840-190o). Pionniers, nègres ou épiciers des lettres? Montréal, Triptyque. $510 \mathrm{p}$.

Michon, Jacques (sous la direction de). 2004. Histoire de l'édition littéraire au Québec au XXe siècle. Le temps des éditeurs. 19401959. Montréal, Fides. 533 p.

Michon, Jacques (sous la direction de). 1994. L'Édition littéraire en quête d'autonomie. Albert Lévesque et son temps. Québec, Presses de l'Université Laval. 214 p.

Pennac, Daniel. 1989. La Petite Marchande de prose. Paris, Gallimard. 403 p.

Ricard, André et Pilon, Jean-Guy. 1991. Les Risques du métier. Montréal, l'Hexagone. $133 \mathrm{p}$.

Ricard, François. 1996. Gabrielle Roy. Une vie. Montréal, Boréal, $642 \mathrm{p}$.

Robert, Lucie. 1989. L'Institution du littéraire au Québec. Sainte-Foy, Presses de l'Université Laval. $272 \mathrm{p}$.

Roy, Gabrielle. 1978. "Comment j'ai reçu le Femina », in Fragiles lumières de la terre. Montréal, Quinze, p. 179-190.

Royer, Jean. 1978. «La vie littéraire. Gabrielle Roy humiliée». Le Devoir, 28 avril, p. 18.

Santantonios, Laurence. 20oo. Auteur/Éditeur. Création sous influence. Enquête. Paris, Loris Talmart. 275 p.

Stanké, Alain. 1989. Occasions de bonheur. Montréal, Les éditions internationales Alain Stanké. 410 p.

Toussaint, Ismène. 2004. Les Chemins retrouvés de Gabrielle Roy. Témoins d'occasions au Québec. Montréal, Les éditions internationales Alain Stanké. 528 p.

Undseld, Siegfried. 1983. L'Auteur et son éditeur. Paris, Gallimard. $263 \mathrm{p}$. 